\title{
Frequency Modulation on Single Sideband Using Controlled Dynamics of an Optically Injected Semiconductor Laser
}

\author{
Sze-Chun Chan, Student Member, IEEE, and Jia-Ming Liu, Senior Member, IEEE
}

\begin{abstract}
Nonlinear dynamics of an optically injected semiconductor laser is applied to photonic microwave generation. By properly adjusting the injection conditions, the optical frequency of the slave laser is first locked to the master laser. The slave laser is then driven into a periodic dynamical state, resulting in a singlesideband (SSB) microwave modulation on the optical carrier. The frequency of the SSB can be controlled by the optical injection strength and detuning. Frequency-modulated SSB can, thus, be obtained from a modulated injection. In this work, we experimentally investigate the generated SSB in terms of its broad tunability and fast modulation response. The results suggest application of this system in radio-over-fiber and optical subcarrier multiplexing technologies when microwave frequency modulation or frequencyshift keying is employed.
\end{abstract}

Index Terms-Dynamics, frequency modulation, injection locked oscillators, microwave generation, semiconductor lasers, subcarrier multiplexing.

\section{INTRODUCTION}

$\mathbf{O}$ PTICALLY injected semiconductor lasers have recently been used in photonic microwave generation [1]. In a master-slave configuration with a sufficient injection power, the optical frequency of the slave laser is injection locked to that of the master laser. By properly adjusting the injection strength and the frequency detuning, instability occurs through Hopf bifurcation into the period-one dynamical state. The result is a tunable microwave oscillation in the optical power of the slave laser [2]. This method of photonic microwave generation offers many advantages due to its single-sideband (SSB) spectrum, broad-frequency tuning range, and ease of stabilization.

In radio-over-fiber systems, optical waves with microwave modulation are used to carry microwave signals over long distance through optical fibers [3]. An SSB spectrum is desirable in such systems because it avoids the microwave power penalty that is caused by the chromatic dispersion [4]. While SSB modulation can be realized by tailoring external electrooptic modulators [5] or by filtering intensity-modulated light [4], [6], the optical injection system described in this paper requires no expensive external components. The microwave modulation generated by our system is naturally SSB, due to the detuned cavity effect associated with the linewidth enhancement factor of a semiconductor laser.

Manuscript received February 7, 2006; revised March 20, 2006

The authors are with the Electrical Engineering Department, University of California, Los Angeles, CA 90095-1594 USA (e-mail: scchan@ucla.edu).

Digital Object Identifier 10.1109/JQE.2006.876712
The optical injection system is also capable of generating high and broadly tunable microwave frequencies of large modulation depths. While the frequency obtained from the direct current modulation on a free-running laser is limited by its relaxation resonance frequency, the nonlinear dynamical oscillations of an optically injected laser reaches much higher frequencies [2]. The all-optical system also bypasses the stringent requirements of high-speed modulation electronics. Frequencies of up to $100 \mathrm{GHz}$ have been observed in our system.

In addition, the optical injection system can readily be stabilized. For both the injected light and the generated SSB light exist inside the slave laser, their microwave beat frequency can be stabilized through various microwave locking techniques, such as self-injection locking [7], [8], external-injection locking [1], and phase locking [9]. These techniques cannot be directly applied if the microwave is instead generated by heterodyning two independent lasers.

Besides, the nonlinear dynamics of semiconductor lasers are conveniently applicable to generating a variety of microwave waveforms [10]. Optically injected semiconductor lasers generate different kinds of periodic waveforms [1], [11] and even broadband chaotic waveforms [12]. Switching among these waveforms can be realized by varying the laser operating conditions. Such waveform diversity is not shared by any other systems of comparable simplicity.

In this paper, we experimentally address the characteristics of the microwave signal generated by an optically injected semiconductor laser. While most of the related studies are conducted on the static characteristics of the microwave generation [13]-[15], many microwave photonics applications require the ability of modulating the microwave signal [16]. For instance, the optical subcarrier multiplexing systems that transmit data on the microwave subcarriers would require such capability [17]-[19]. Therefore, we focus on the dynamic modulation characteristics of the microwave generated under a modulated optical injection. A fast dynamical response is measured, which shows the potential of this system for communication applications. Following this introduction, the experimental setup is described in Section II. The static tuning characteristics are reported in Section III, while the dynamic characteristics are examined in Section IV. It is then followed by a discussion and a conclusion in Section V and Section VI, respectively.

\section{EXPERIMENTAL SETUP}

A simplified schematic of the experimental setup is shown in Fig. 1(a). The semiconductor lasers used are $1.3-\mu \mathrm{m}$ 


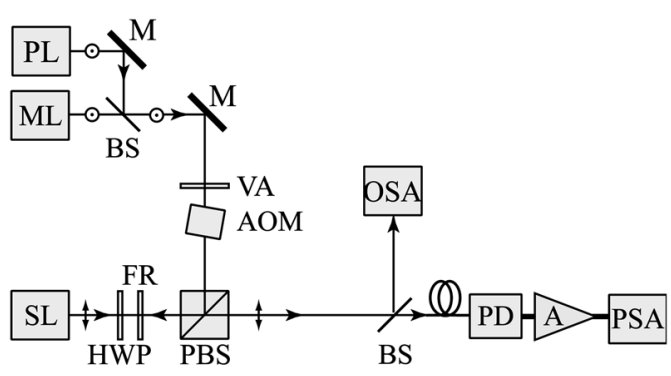

(a)

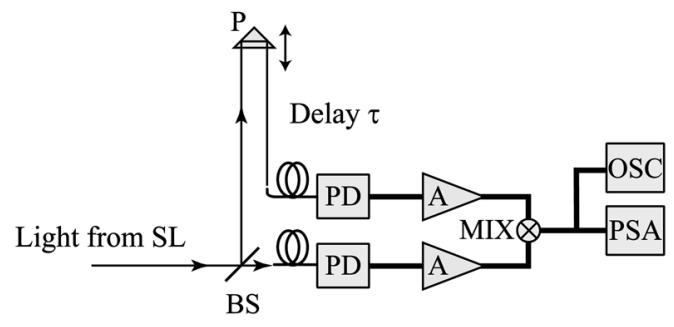

(b)

Fig. 1. Schematics of the experimental setup. (a) Optical injection system. (b) Microwave homodyne detection. ML: master laser; SL: slave laser; PL: probe laser; M: mirror; BS: beam splitter; VA: variable attenuator; AOM: acoustooptic modulator; PBS: polarizing beam splitter; FR: Faraday rotator; HWP: half-wave plate; P: prism; PD: fiber-coupled photodiode; A: amplifier; MIX: mixer; PSA: power spectrum analyzer; OSC: oscilloscope; and OSA: optical spectrum analyzer.

single-mode distributed feedback lasers (Bookham Technology LC131). The slave laser is biased at 2.22 times its $18-\mathrm{mA}$ threshold and temperature stabilized at $18.00^{\circ} \mathrm{C}$. At an output power of $4.5 \mathrm{~mW}$, it has a relaxation resonance frequency of about $10 \mathrm{GHz}$ and a linewidth enhancement factor of about 3.2. Light is injected into the slave laser from a master laser through a free-space circulator arrangement that consists of a polarizing beam splitter, a Faraday rotator, and a half-wave plate. The optical frequency of the master laser is tuned to be the same as that of the slave laser and $7.3 \mathrm{~mW}$ of light is injected onto the front facet of the slave laser. The injection forces the slave laser into a period-one dynamical state, where its optical power oscillates at a microwave frequency $f_{0}$ of 15.24 GHz. This operating point is regarded as the bias point upon which modulation is to be applied. The output from the slave is split into two by a beam splitter such that its optical and power spectra are monitored simultaneously. The optical spectrum is monitored by an optical spectrum analyzer (Newport SuperCavity SR-260-C), and the power spectrum is monitored by a $26.5-\mathrm{GHz}$ power spectrum analyzer (HP E4407B) after a photodiode and an amplifier.

Both the frequency and the amplitude of the microwave oscillation depend on the parameters of the optical injection. The injection field can be adjusted by a variable attenuator or intensity-modulated by an acoustooptic modulator (AOM) (Brimrose AMF-100-1300). A modulated injection can be realized by adding a small amount of light from a probe laser, which is operated at about the same optical frequency as the master laser. The light from the probe laser can be regarded as a sideband

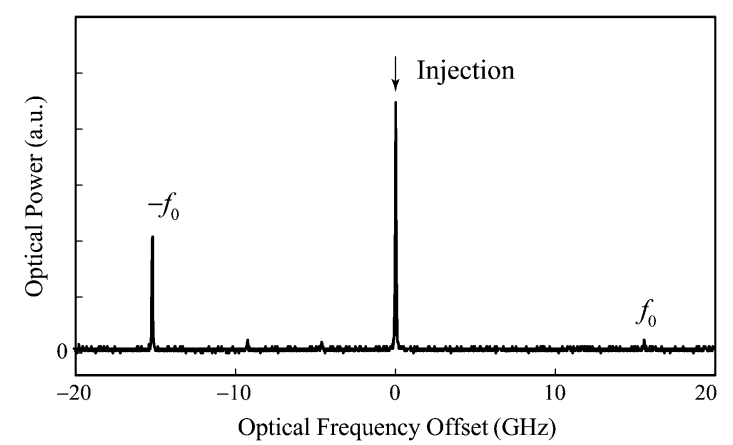

Fig. 2. Optical spectrum of the slave laser under steady-state period-one oscillation. The frequency axis is offset to the free-running frequency of the slave laser. The arrow indicates the injection from the master laser, which is at zero detuning from the slave laser. The sidebands are due to the period-one oscillation at $f_{0}=15.24 \mathrm{GHz}$.

of the light from the master laser. The frequency difference between the master laser and the probe laser is equivalent to the modulation frequency of the injection.

As a result of the modulated injection, the period-one state of the slave laser is modulated correspondingly. The microwave oscillation of the period-one state is generally under both frequency modulation (FM) and amplitude modulation (AM). It is analyzed using a delayed microwave (not optical) homodyne method as shown in Fig. 1(b). The optical output of the slave laser is divided into two arms, each consisting of a photodiode and an amplifier, which have a combined bandwidth of 6-18 GHz. The amplified signals, each of about $-2.5 \mathrm{dBm}$, are sent into a mixer (WJ MV50C) that is monitored by the power spectrum analyzer or a 3-GHz real-time oscilloscope (Tektronix TDS694C). By properly adjusting the relative delay time $\tau$ using the prism, the FM and the AM components of the modulated microwave signal can be analyzed separately.

\section{STATIC Characteristics}

In this section, the steady-state characteristics of the period-one oscillation are investigated. The probe laser is blocked and the AOM is switched off during the experiment. The optical frequency detuning of the master laser to the free-running slave laser is set at zero. The master laser then injects $7.3 \mathrm{~mW}$ of the optical power to the slave laser. Fig. 2 shows the optical spectrum offset to the free-running frequency of the slave laser. The slave laser is injection-locked to the master laser, resulting in the frequency component at the zero frequency offset as indicated by the arrow. Due to the laser nonlinear dynamics, the relaxation oscillation of the slave laser is modified and undamped by the injection [20]. Sidebands separated from the injection by $f_{0}=15.24 \mathrm{GHz}$ are generated. (Ignore the small peaks at -4.5 and $-9 \mathrm{GHz}$, which are artifacts from the high-order transverse modes of the Fabry-Perot optical spectrum analyzer). Note that the sidebands are highly asymmetric, which is also observed in a related study [13].

The asymmetry can be explained by the effect of the cavity resonance of the slave laser under the influence of the antiguidance effect, which is governed by the linewidth enhancement factor [20], [21]. With the input of the optical field from the injection, the gain that must be maintained in the slave laser is 


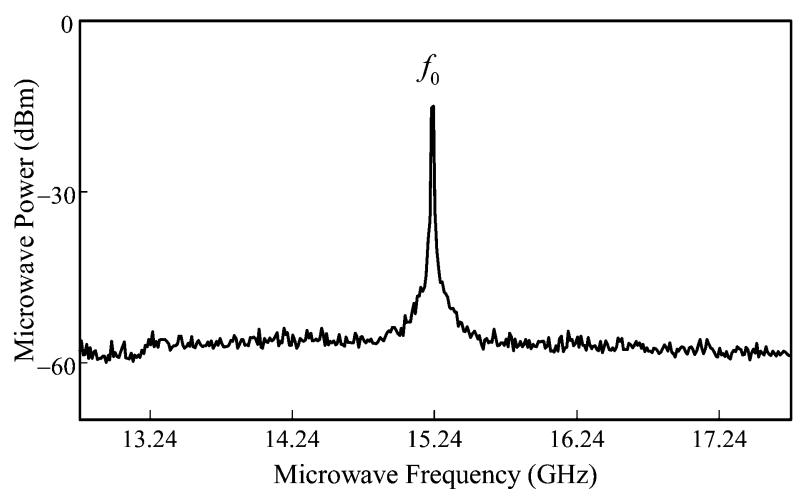

Fig. 3. Power spectrum of the slave laser under steady-state period-one oscillation of $f_{0}=15.24 \mathrm{GHz}$ (resolution bandwidth $=3 \mathrm{MHz}$ ).

reduced. The gain reduction implies a decrease in the chargecarrier density. Through the antiguidance effect, the refractive index of the gain medium increases accordingly. The effective cavity length, thus, increases, which lowers the cavity resonance frequency. Therefore, the negative sideband of the period-one state is significantly enhanced by the red-shifted cavity resonance. This causes a nearly SSB spectrum that consists mainly of the injected frequency component and the lower sideband of the period-one oscillation. The SSB behavior is beneficial to the microwave signal distribution in radio-over-fiber systems. It avoids the dispersion-induced RF power penalty, which is often experienced by a double-sideband signal.

Fig. 3 shows the microwave signal at $f_{0}=15.24 \mathrm{GHz}$ that is generated by the beating of the optical frequencies inside the photodiode. The spontaneous emission noise and the chargecarrier fluctuations result in a microwave linewidth on the order of $10 \mathrm{MHz}$. Although it is not the intent of this paper to stabilize the microwave signal, it should be mentioned that several microwave injection-locking techniques have been demonstrated in significantly reducing the microwave linewidth [1], [7]-[9].

The generated microwave signal is also widely tunable. When the frequency detuning of the injection is kept at zero, a range of $f_{0}$ can be obtained by varying the injection strength. Fig. 4(a) shows the nearly linear dependence of the required injection electric field amplitude on the desired frequency of the microwave signal, while Fig. 4(b) shows the corresponding microwave power obtained. The microwave power emerges at about $f_{0}=11.3 \mathrm{GHz}$, close to the original relaxation resonance frequency of the slave laser, and it stays almost constant as the frequency increases. Therefore, the generation of a widely tunable microwave frequency that circumvents the usual modulation bandwidth limitation of the laser is achieved.

The microwave signal can also be tuned by varying the frequency detuning of the injection. Fig. 5(a) shows the required detuning as a function of the desired $f_{0}$ under a fixed injection power of $7.3 \mathrm{~mW}$. The generated $f_{0}$ increases as the detuning increases. The corresponding microwave power generated is shown in Fig. 5(b). The power stays nearly constant as long as the slave laser is in the period-one state, which occurs for $f_{0}>14.8 \mathrm{GHz}$.

These results show that the system can be used as an optically controlled microwave source of large tunability. With a proper
Zero injection frequency detuning
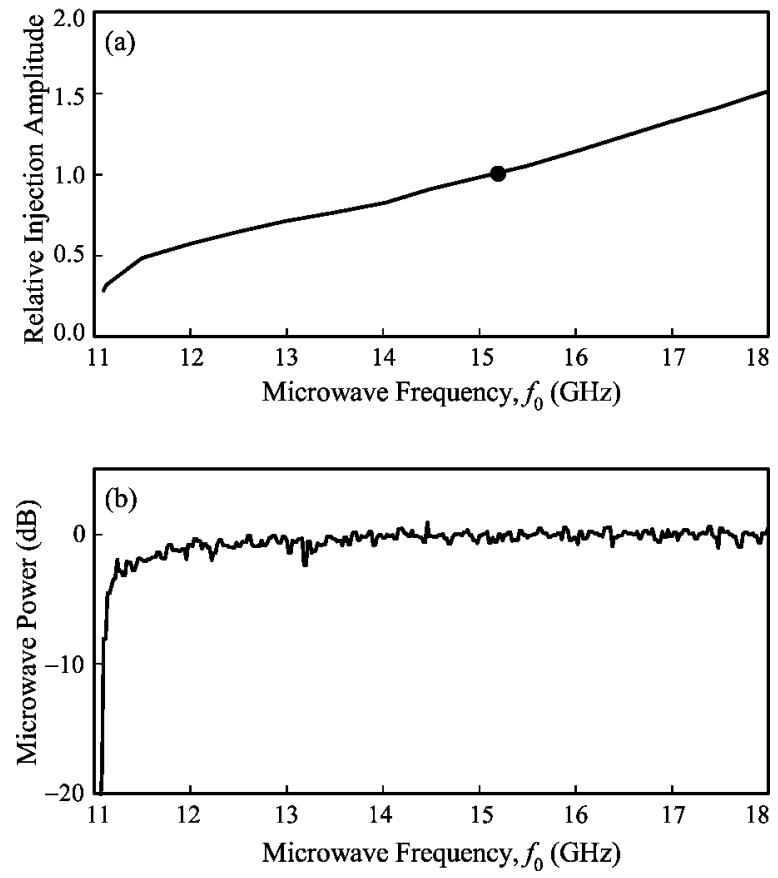

Fig. 4. Tuning characteristics for generating microwave frequency $f_{0}$ under injection at zero detuning. (a) The relative injection field amplitude required. (b) The relative microwave power generated.

\section{3-mW injection}
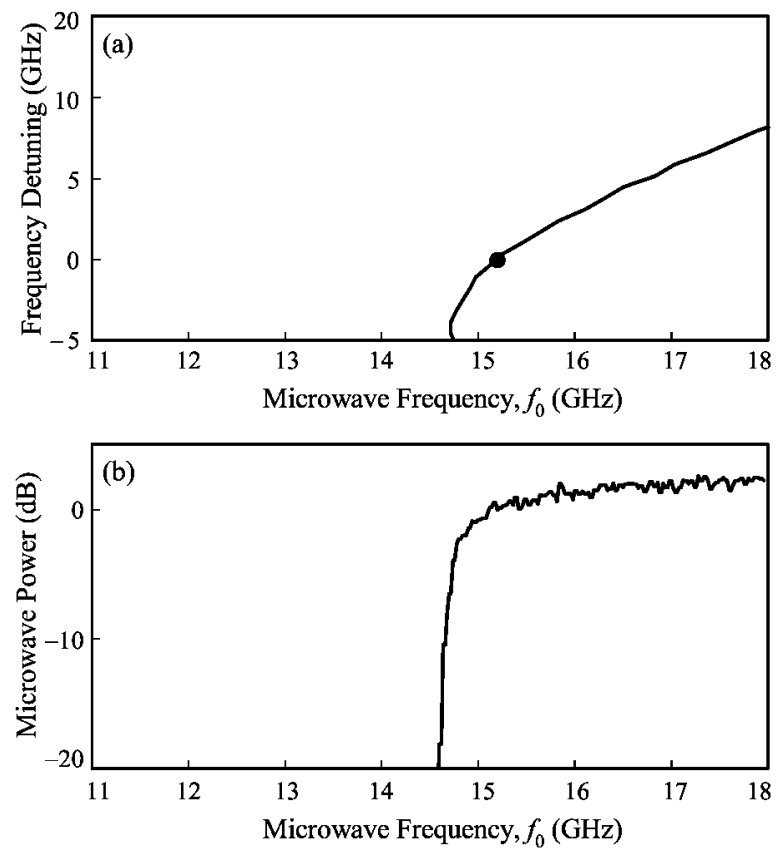

Fig. 5. Tuning characteristics for generating microwave frequency $f_{0}$ under a constant $7.3-\mathrm{mW}$ injection. (a) The injection frequency detuning required. (b) The relative microwave power generated.

combination of the injection strength and detuning, frequencies of up to $100 \mathrm{GHz}$ are observed experimentally. However, due to the bandwidth limitation of the available detection electronics, we focus our studies in this paper to the period-one state at a lower frequency. Under a $7.3-\mathrm{mW}$ injection at zero detuning, 
a period-one state of $f_{0}=15.24 \mathrm{GHz}$ is obtained. This operating point is common to both Figs. 4 and 5, as indicated by the solid dots in both figures. In Section IV, the operating point is regarded as a bias point, around which modulation is applied to study the dynamic characteristics of the period-one state.

\section{DYNAMIC CHARACTERISTICS}

The dynamic characteristics of the period-one state are investigated in detail by using a modulated injection. The weak probe laser is unblocked and its output is combined with that of the master laser into the injection path. By regarding the light from the probe laser as a sideband of the light from the master laser, the injection is SSB modulated at a modulation frequency $f_{m}$, where $f_{m}$ equals the optical frequency difference between the probe laser and the master laser. The modulation depth and frequency can be respectively varied by adjusting the power and frequency of the probe laser. As an extension of the static tuning, the modulated injection is expected to result in a modulation on the frequency and amplitude of the period-one oscillation.

Initially, the probe laser is tuned so as to obtain a slow modulation of $f_{m}=10 \mathrm{MHz}$. The relative power of the probe laser to that of the master laser is varied from 0 to 0.01 , which corresponds to an equivalent modulation depth on the injection electric field in the range of 0 to 0.10 . The subsequently modulated period-one state is monitored as shown in Fig. 6. The optical spectra are shown in the left column for the modulation depths of $0.05,0.08$, and 0.10 in Fig. 6(a-i), (b-i), and (c-i), respectively. Though the modulation sideband of the injection itself is too weak to be observed, its effect shows as a progressive broadening of the generated peak at $-f_{0}$. In fact, the power spectrum shown in the right column gives clear evidence of FM on $f_{0}$ around the center frequency of $15.24 \mathrm{GHz}$. The maximum frequency deviation, denoted by $\Delta f$, increases with the injection modulation depth. It is measured to be $0.10,0.35$, and $0.60 \mathrm{GHz}$ for Fig. 6(a-ii), (b-ii), and (c-ii), respectively.

The behavior of the period-one state at a few different modulation frequencies is then investigated. The modulation depth of the injection is kept constant at 0.10 . By tuning the frequency of the probe laser, the optical and the power spectra of the period-one state at different values of $f_{m}$ are obtained, as shown in Fig. 7. Fig. 7(a-i) shows the optical spectrum under $f_{m}=$ $80 \mathrm{MHz}$. The peak at $-f_{0}$ is again broadened as a result of the FM. The corresponding power spectrum in Fig. 7(a-ii) shows a continuum of frequencies with a maximum deviation of $\Delta f=$ $0.65 \mathrm{GHz}$. Because $f_{m}<\Delta f$, the state is said to be under a wideband FM [22]. Although the spectrum under the periodic modulation ought to contain discrete peaks separated by $f_{m}$, the separation is too small to be resolved in Fig. 7(a-ii).

As $f_{m}$ increases to $500 \mathrm{MHz}$, the discreteness becomes apparent. The optical spectrum in Fig. 7(b-i) shows that a sharp peak at $-f_{0}$ is now accompanied by closely separated sidebands. The sidebands are more clearly shown in the power spectrum of Fig. 7(b-ii) as peaks separated by multiples of $f_{m}$ from $f_{0}$. The discreteness of the spectrum hinders an accurate determination of $\Delta f$ because it is now comparable to $f_{m}$.

When $f_{m}$ is increased to $2 \mathrm{GHz}$, the modulation sidebands of $-f_{0}$ are too small to be recorded by the optical spectrum
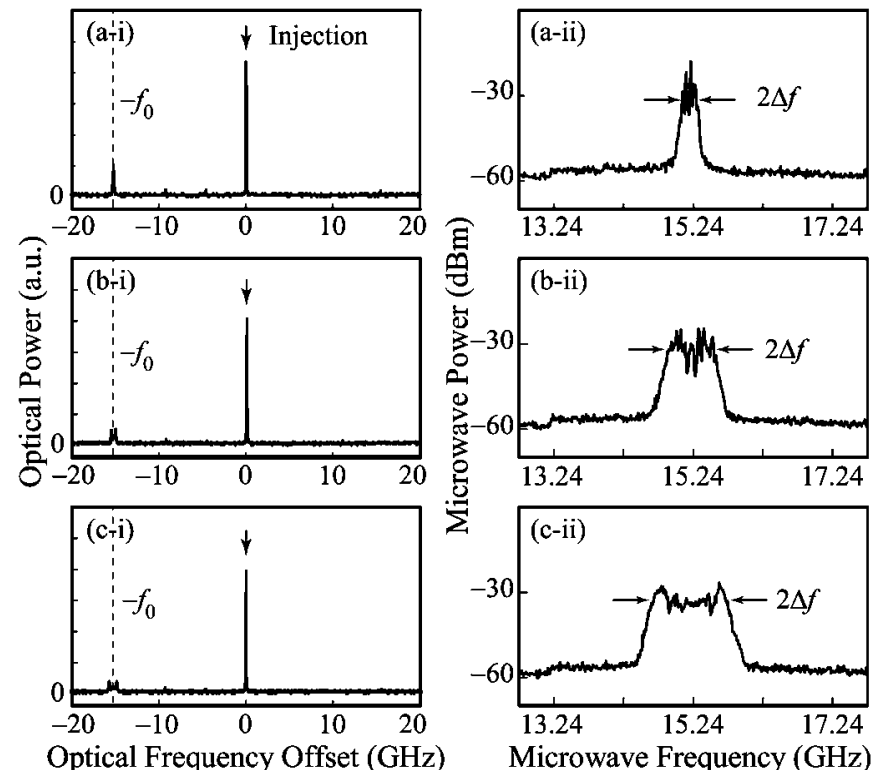

Fig. 6. Behavior of the modulated period-one oscillation under a slowly modulated injection $\left(f_{m}=10 \mathrm{MHz}\right.$ ). (i) Optical spectrum. (ii) Power spectrum. The modulation depths of the injection field amplitude are: (a) 0.05, (b) 0.08, and (c) 0.10 .
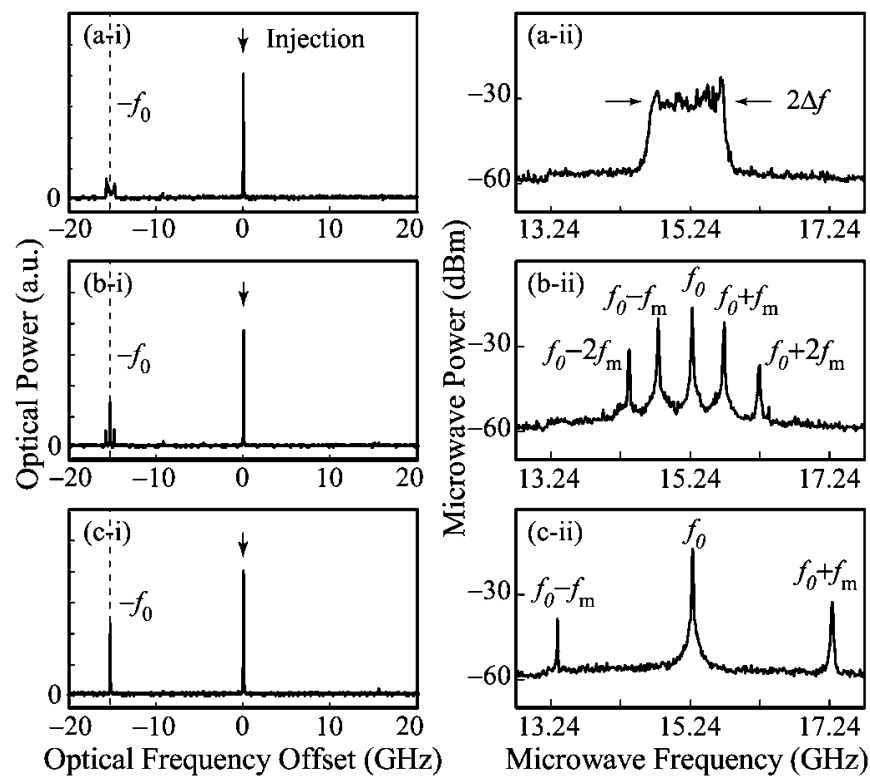

Fig. 7. Behavior of the period-one oscillation under a modulated injection with a depth of 0.10. (i) Optical spectrum. (ii) Power spectrum. The modulation frequency $f_{m}$ is: (a) $80 \mathrm{MHz}$, (b) $500 \mathrm{MHz}$, and (c) $2 \mathrm{GHz}$.

in Fig. 7(c-i), but they are observed in the power spectrum in Fig. 7(c-ii). Because $f_{m}>\Delta f$, the spectrum belongs to that of a narrowband FM. The determination of $\Delta f$ needs to rely on a time-domain method as outlined below.

In general, the modulated microwave signal generated after the photodiode can be represented by

$$
V(t)=\left(V_{0}+v(t)\right) e^{-i 2 \pi f_{0} t+i \phi(t)}+\text { c.c. }
$$



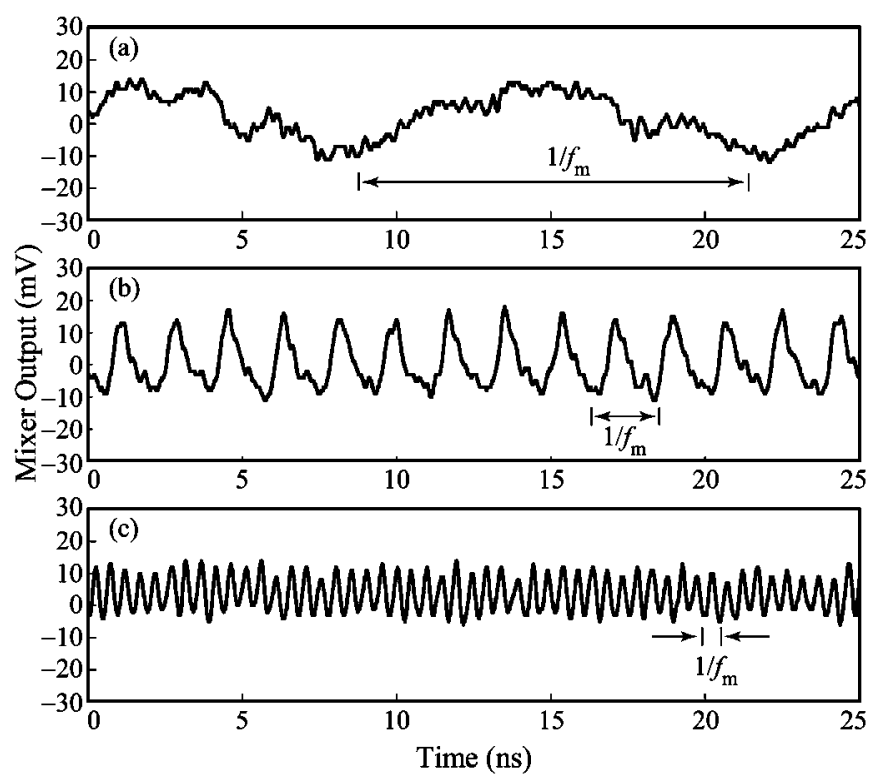

Fig. 8. Mixer output with $\tau=\left(4 f_{0}\right)^{-1}$ measuring the FM through a conversion factor of $-50 \mathrm{MHz} / \mathrm{mV}$. The modulation frequency $f_{m}$ is: (a) $80 \mathrm{MHz}$, (b) $500 \mathrm{MHz}$, and (c) $2 \mathrm{GHz}$.

where $V_{0}$ is the steady-state amplitude of the microwave generated, $v(t)$ is the AM part of the signal, and $\phi(t)$ is the instantaneous phase due to FM. Under a modulation at $f_{m}$, the instantaneous microwave frequency is given by

$$
f_{0}-\frac{1}{2 \pi} \frac{d \phi}{d t}=f_{0}+\Delta f \cos \left(2 \pi f_{m} t\right)
$$

The AM part can be written as

$$
v(t)=m V_{0} \cos \left(2 \pi f_{m} t+\alpha\right)
$$

where $m$ and $\alpha$ are, respectively, the index and the phase of the AM.

In order to determine $\Delta f$ for the whole range of $f_{m}$, a delayed microwave homodyne method is applied [Fig. 1(b)]. The mixer output is, thus

$$
\begin{aligned}
M=[ & \left.\left(V_{0}+v(t)\right) e^{-i 2 \pi f_{0} t+i \phi(t)}\right] \\
& \times\left[\left(V_{0}+v(t-\tau)\right) e^{-i 2 \pi f_{0}(t-\tau)+i \phi(t-\tau)}\right]^{*}+\text { c.c. }
\end{aligned}
$$

The delay is set to be $\tau=\left(4 f_{0}\right)^{-1}$ so that

$$
M \approx-4 \pi V_{0}^{2} \tau \Delta f \cos \left(2 \pi f_{m} t\right)
$$

The mixer output is, thus, modulated at the modulation frequency $f_{m}$, and its amplitude measures the maximum frequency deviation $\Delta f$ of the FM. Fig. 8(a)-(c) shows the mixer output at $f_{m}=80,500$, and $2 \mathrm{GHz}$, respectively. The amplitudes of these traces, after a conversion factor of $-50 \mathrm{MHz} / \mathrm{mV}$, gives $\Delta f$ of $0.65,0.60$, and $0.50 \mathrm{GHz}$, respectively. The variation of $\Delta f$ with respect to $f_{m}$ is the frequency response of the FM. The

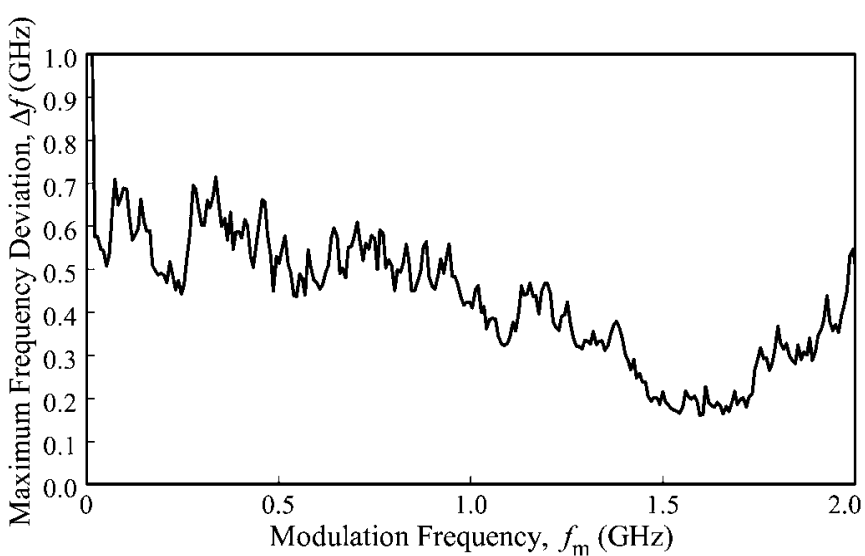

Fig. 9. FM frequency response of the period-one state.

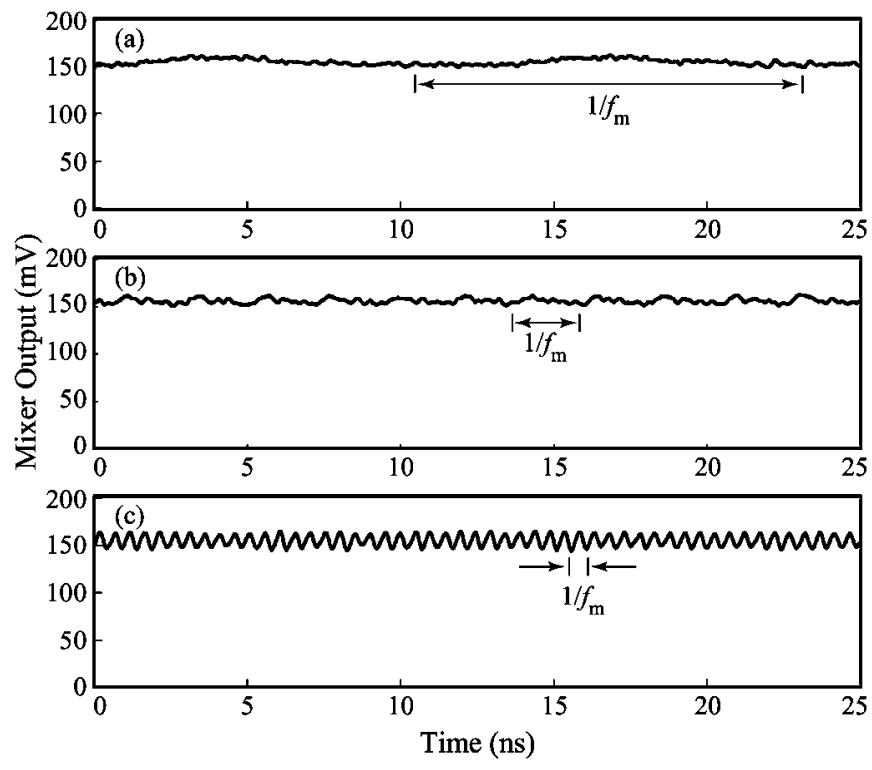

Fig. 10. Mixer output with $\tau=0$ measuring the AM. The modulation frequency $f_{m}=$ (a) $80 \mathrm{MHz}$, (b) $500 \mathrm{MHz}$, and (c) $2 \mathrm{GHz}$.

FM frequency response under an injection modulation depth of 0.10 is shown in Fig. 9, where a broad modulation bandwidth is observed. The maximum frequency deviation $\Delta f$ is about $0.6 \mathrm{GHz}$ at $f_{m}=0$, which agrees with the static measurement in Fig. 4(a) when the injection amplitude changes by $10 \%$ around the bias point.

The modulated period-one oscillation is predominantly under FM, but the AM response is also measured for completeness. The delay is set at $\tau=0$ so that

$$
M \approx 2 V_{0}^{2}\left(1+2 m \cos \left(2 \pi f_{m} t+\alpha\right)\right)
$$

which undulates with an amplitude proportional to $m$. Fig. 10(a)-(c) shows the mixer output again at $f_{m}=80$, 500 , and $2 \mathrm{GHz}$, respectively. From the very small undulation amplitude, the AM index $m$ is determined to be $0.020,0.012$, and 0.035 , respectively. The AM frequency response is summarized in Fig. 11, which shows a small $m$ over the measured frequency range. 


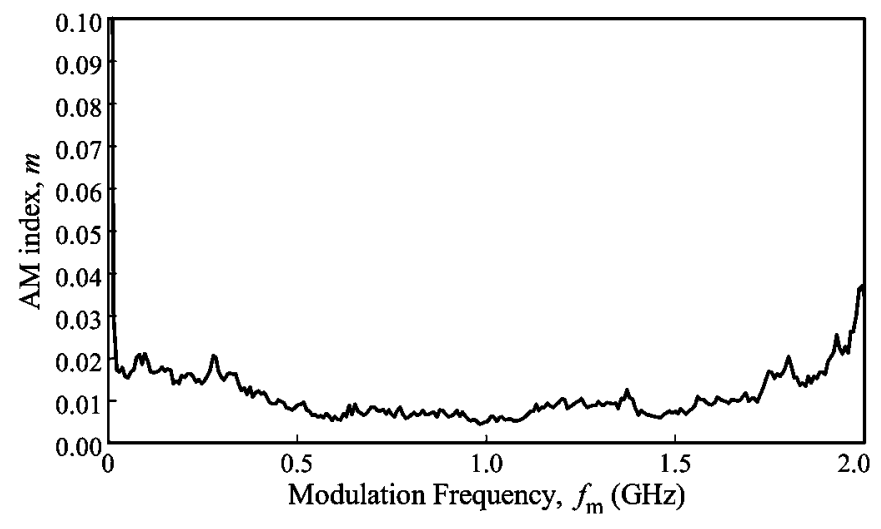

Fig. 11. AM frequency response of the period-one state.

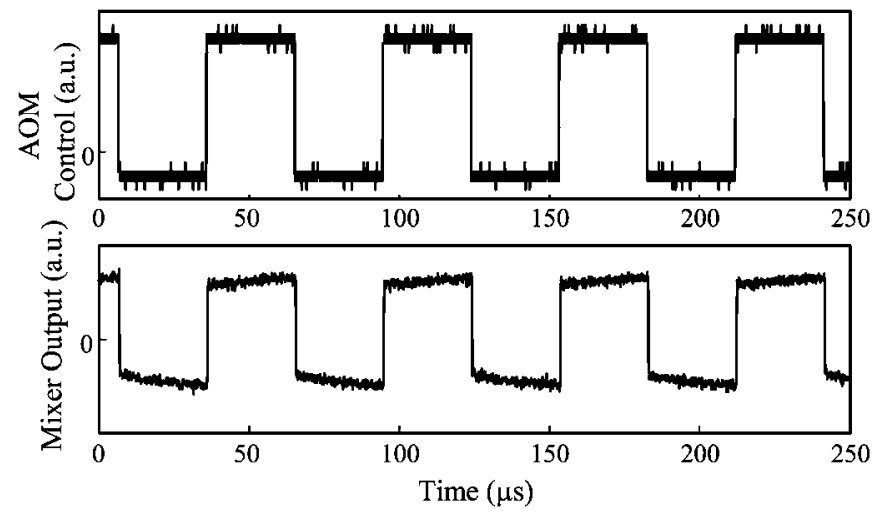

Fig. 12. Frequency switching of the period-one state.

All the experimental data suggest that the period-one state can be easily frequency modulated. With the broad frequency response and the small residual AM component, the optical injection scheme is applicable to FM communication systems. The laser output can be used to carry, over long optical fibers, a high-frequency microwave subcarrier $f_{0}$, which is frequency modulated by broadband data.

\section{DISCUSSION}

Thus far, the experiments are on the analog modulation response of the period-one state. However, applications such as frequency-shift keying require digitally switching the state from one $f_{0}$ to another. A switched injection that carries digital data or codes can be used to switch $f_{0}$ in these scenarios. With the probe laser being blocked, the frequency switching is demonstrated by digitally controlling the AOM. When the control signal is on, a small portion of the injection power is diffracted astray from the injection path. When the control signal is off, the injection is unaffected by the AOM. The resultant switching of $f_{0}$ is again monitored through delayed homodyning. It is shown in Fig. 12. Frequency switching between 15.26 and $14.87 \mathrm{GHz}$ is confirmed from the microwave spectrum. This shows the feasibility for digital applications. The data rate in our demonstration is currently limited by the speed of the AOM. However, the broad FM frequency response of the period-one state in Fig. 9 suggests that the optical injection system can support a much higher data rate of up to at least
$1 \mathrm{GHz}$. Experiments are conducted by directly modulating the master laser, instead of the AOM, using an OC-12 622-Mbps data stream. The digital AM output from the master laser is then converted by the slave laser into a digital FM signal. Detailed results will be presented in a future publication.

Our system can be compared to the experiment by Kaszubowska et al. [16], where the period-one state is also used for radio-over-fiber communication. The data modulation is first applied on an external microwave source, and the data-carrying microwave is then used to injection-lock the slave laser. Injection-locking works by the pulling of the original period-one frequency. It forces the laser to oscillate according to the external microwave. The maximum frequency deviation $\Delta f$ of such a system is, thus, limited by the locking range of the period-one state. In contrast, our system is based on the modulation of the period-one frequency by instead controlling the laser dynamics. Hence, our $\Delta f$ is limited only by the tuning range of the period-one state, which we have shown to be rather broad (Fig. 4).

Although our dynamical characterization was done only on one period-one state, similar results are observed for the other period-one states as well. The sensitivity of the microwave frequency to the injection modulation is expected to vary slightly, according to the variation of the local slopes on the tuning curves in Figs. 4(a) and 5(a). Therefore, using an optimal period-one state would result in an improved FM performance. Similar to the numerical study on the static characteristics in [13], a comprehensive investigation can be conducted on the dynamical characteristics, so as to extract their dependence on the operating point and the laser dynamical parameters. Theoretical studies are also necessary in order to verify the FM frequency response measurements and to understand the mechanisms behind a modulated period-one state.

\section{CONCLUSION}

We have investigated the characteristics of an optically injected semiconductor laser. The controlled nonlinear laser dynamics of the period-one oscillation state is used to generate microwave signals. The microwave generated is widely tunable, and it tends to appear as an SSB modulation on the optical carrier, which is desirable for efficient transmission of microwave signals over optical fibers. By modulating the optical injection, the behavior of the modulated period-one state is examined for the first time. The state is easily frequency modulated, and a broad FM bandwidth is measured. The results show that the optical injection system can be used for FM radio-over-fiber applications.

\section{REFERENCES}

[1] T. B. Simpson and F. Doft, "Double-locked laser diode for microwave photonics applications," IEEE Photon. Technol. Lett., vol. 11, no. 11, pp. 1476-1478, Nov. 1999.

[2] T. B. Simpson, J. M. Liu, K. F. Huang, and K. Tai, "Nonlinear dynamics induced by external optical injection in semiconductor lasers," Quantum Semiclass. Opt., vol. 9, pp. 765-784, Oct. 1997.

[3] C. Lim, A. Nirmalathas, D. Novak, R. Waterhouse, and G. Yoffe, "Millimeter-wave broad-band fiber-wireless system incorporating baseband data transmission over fiber and remote LO delivery," $J$. Lightw. Technol., vol. 18, no. 10, pp. 1355-1363, Oct. 2000. 
[4] H. S. Ryu, Y. K. Seo, and W. Y. Choi, "Dispersion-tolerant transmission of $155-\mathrm{Mb} / \mathrm{s}$ data at $17 \mathrm{GHz}$ using a $2.5-\mathrm{Gb} / \mathrm{s}$-grade DFB laser with wavelength-selective gain from an FP laser diode," IEEE Photon. Technol. Lett., vol. 16, no. 8, pp. 1942-1944, Aug. 2004.

[5] D. Novak, G. H. Smith, A. J. Lowery, H. F. Liu, and R. B. Waterhouse, "Millimetre-wave fibre-wireless transmission systems with reduced effects of fibre chromatic dispersion," Opt. Quantum Electron., vol. 30, pp. 1021-1031, Dec. 1998.

[6] A. Kaszubowska, P. Anandarajah, and L. P. Barry, "Multifunctional operation of a fiber Bragg grating in a WDM/SCM radio over fiber distribution system," IEEE Photon. Technol. Lett., vol. 16, no. 2, pp. 605-607, Feb. 2004.

[7] P. Saboureau, J. P. Foing, and P. Schanne, "Injection-locked semiconductor lasers with delayed optoelectronic feedback," IEEE J. Quantum Electron., vol. 33, no. 9, pp. 1582-1591, Sep. 1997.

[8] S. C. Chan and J. M. Liu, "Tunable narrow-linewidth photonic microwave generation using semiconductor laser dynamics," IEEE J. Sel. Topics Quantum Electron., vol. 10, no. 5, pp. 1025-1032, Sep./Oct. 2004.

[9] T. B. Simpson, "Phase-locked microwave-frequency modulations in optically-injected laser diodes," Opt. Commun., vol. 170, pp. 93-98, Oct. 1999.

[10] F. Y. Lin and J. M. Liu, "Diverse waveform generation using semiconductor lasers for radar and microwave applications," IEEE J. Quantum Electron., vol. 40, no. 6, pp. 682-689, Jun. 2004.

[11] S. C. Chan and J. M. Liu, "Microwave frequency division and multiplication using an optically injected semiconductor laser," IEEE J. Quantum Electron., vol. 41, no. 9, pp. 1142-1147, Sep. 2005.

[12] J. M. Liu, H. F. Chen, and S. Tang, "Optical-communication systems based on chaos in semiconductor lasers," IEEE Trans. Circuits Syst. I, Fundam. Theory Appl., vol. 48, no. 12, pp. 1475-1483, Dec. 2001.

[13] S. K. Hwang, J. M. Liu, and J. K. White, "Characteristics of period-one oscillations in semiconductor lasers subject to optical injection," IEEE J. Sel. Topics Quantum Electron., vol. 10, no. 5, pp. 974-981, Sep./Oct. 2004.

[14] T. Erneux, V. Kovanis, A. Gavrielides, and P. M. Alsing, "Mechanism for period-doubling bifurcation in a semiconductor laser subject to optical injection," Phys. Rev. A., vol. 53, pp. 4372-4380, Jun. 1996.

[15] M. Nizette, T. Erneux, A. Gavrielides, and V. Kovanis, "Stability and bifurcations of periodically modulated, optically injected laser diodes," Phys. Rev. E., vol. 63, pp. 026212-026212, Feb. 2001.

[16] A. Kaszubowska, L. P. Barry, and P. Anandarajah, "Multiple RF carrier distribution in a hybrid radio/fiber system employing a self-pulsating laser diode transmitter," IEEE Photon. Technol. Lett., vol. 14, no. 11, pp. 1599-1601, Nov. 2002.

[17] X. L. Wang, G. F. Li, and C. S. Ih, "Microwave/millimeter-wave frequency subcarrier lightwave modulations based on self-sustained pulsation of laser diode," J. Lightw. Technol., vol. 11, no. 2, pp. 309-315, Feb. 1993.

[18] R. P. Braun, G. Grosskopf, H. Heidrich, C. von Helmolt, R. Kaiser, K. Kruger, U. Kruger, D. Rohde, F. Schmidt, R. Stenzel, and D. Trommer, "Optical microwave generation and transmission experiments in the 12- and 60-GHz region for wireless communications," IEEE Trans. Microw. Theory Tech., vol. 46, no. 4, pp. 320-330, Apr. 1998.
[19] F. N. Timofeev, S. Bennett, R. Griffin, P. Bayvel, A. J. Seeds, R. Wyatt, R. Kashyap, and M. Robertson, "High spectral purity millimetre-wave modulated optical signal generation using fibre grating lasers," Electron. Lett., vol. 34, pp. 668-669, Apr. 1998.

[20] T. B. Simpson, J. M. Liu, and A. Gavrielides, "Small-signal analysis of modulation characteristics in a semiconductor laser subject to strong optical injection," IEEE J. Quantum Electron., vol. 32, no. 8, pp. 1456-1468, Aug. 1996.

[21] A. Gavrielides, V. Kovanis, T. Erneux, and M. Nizette, "Phase locked modulations of optically injected laser diodes," Proc. SPIE, vol. 3944, pp. $627-638,2000$.

[22] S. Haykin, Communication Systems, 3rd ed. New York: Wiley, 1994.

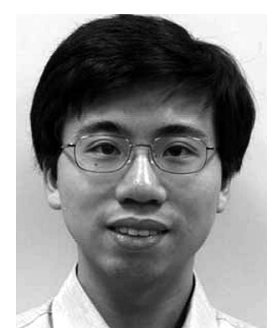

Sze-Chun Chan (S'98) received the B.Eng. degree in electrical and electronic engineering from the University of Hong Kong, in 2001, and the M.S. degree in electrical engineering from the University of California, Los Angeles (UCLA), in 2004. He is currently pursuing the Ph.D. degree in electrical engineering at UCLA.

His current research focuses on the nonlinear dynamics of semiconductor lasers and its photonic microwave applications.

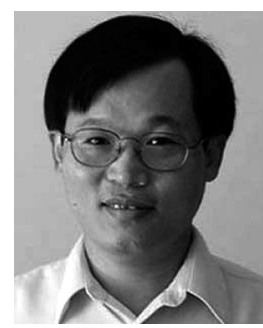

Jia-Ming Liu (M'83-SM'85) received the B.S. degree in electrophysics from the National Chiao Tung University, Taiwan, R.O.C., in 1975, and the M.S. and $\mathrm{Ph} . D$. degrees in applied physics from Harvard University, Cambridge, MA, in 1979 and 1982, respectively.

He became a Licensed Professional Electrical Engineer in 1977. He was an Assistant Professor with the Department of Electrical and Computer Engineering, State University of New York at Buffalo, from 1982 to 1983 , and a Senior Member of the technical staff with GTE Laboratories, Inc., from 1983 to 1986. He is currently Professor of Electrical Engineering at University of California, Los Angeles. His current research interests include development and application of ultrafast wavelength-tunable laser pulses, nonlinear and ultrafast processes in materials and devices, optical wave propagation, optical communications, nonlinear dynamics of lasers, and chaotic communications.

Dr. Liu is a fellow of the Optical Society of America and the American Physical Society, a senior member of the IEEE Laser and Electro-Optics Society, and a founding member of the Photonics Society of Chinese-Americans. 